\title{
ELECTRICAL IMPEDANCE TOMOGRAPHY (EIT) BASED MEDICAL IMAGING USING FINITE ELEMENT METHOD (FEM)
}

\author{
Vidya Sarode ${ }^{1}$, Priya M. Chimurkar ${ }^{2}$, Alice N. Cheeran ${ }^{3}$ \\ ${ }^{1}$ Research Scholar, Xavier Institute of Engineering, Mumbai, India. \\ ${ }^{2 \& 3}$ Electrical Department, VJTI, Mumbai, India.
}

\begin{abstract}
The resistivity of biological tissue happens to be the basis of the field distribution inside the tissue. Hence the current injected in different patterns has a unique current distribution profile. The images of this field distribution are reconstructed using tools like FEMM and EIDORS. The work described here is the EIT based imaging of a closed phantom for detection of abnormality present. This system consists of 16 silver electrodes placed equidistant and detects non-conducting and conducting objects like clay and copper spindle in normal saline solution. The current is injected in two adjacent electrodes and the voltages are measured in neighbouring pattern at every consecutive pair of electrodes. The output data sets are used to reconstruct the voltage density distribution in closed phantom. The resulting images show that the resistivity profiles of the phantom for various events are successfully reconstructed. In this paper, the voltage density distribution is reconstructed into an image to detect the type, the location and the size of the abnormality present using FEMM tool.
\end{abstract}

\section{KEYWORDS}

EIT, Field Distribution, FEMM, Non-conducting impurity, Conducting Impurity.

\section{INTRODUCTION}

EIT (Electrical Impedance Tomography) is a non invasive medical imaging technique in which current is applied to surface of the body through contact electrodes and the resulting surface voltages are used in reconstruction of images. EIT is based on electrical properties of different materials, mainly the conductivity of material which is used in phantom [1, 2, 3]. EIT does not use harmful radiations. It identifies information of electrical properties of tissue [4]. This is a new methodology for use in medical applications. It has the potential to replace the conventional medical imaging techniques. The commonly known medical imaging techniques are Computed Tomography (CT), Mammography, Magnetic Resonance Imaging (MRI), Positron Emission tomography (PET), Ultrasound and Thermal imaging [5]. In all these techniques either harmful radiations are used which may cause side effects to body tissues or the cost is not affordable. Thermography can help in diagnosis of advanced abnormality but has the limitation that the change in surface temperature occurs only if the impurity is near to the surface [6]. Despite of the side effects the above techniques also require the special attention of physicians. Studying the Impedance Image reconstruction with practical phantoms is necessary to assess the performance of the EIT system. Practical phantoms with saline and solid resistive materials are very popular in EIT as they are low cost and easy to develop [7]. EIT is very advantageous due to its cost effectiveness i.e. it is affordable to common man and portability. Also not much expertise is required to conduct this experiment. The experiments are explained in subsequent sections. Section 2 describes the EIT system and the experimental set up. The details of data collection are described in section 3. The reconstructed FEM graphs for two types of 
International Journal of Engineering Sciences \& Emerging Technologies, Feb 2012. ISSN: 2231 - 6604 doi: 10.7323/ijeset/v1_i2_10 Volume 1, Issue 2, pp: 83-89 OIJESET

impurities are explained in section 4 . Section 5 interprets the results. The summary is concluded in section 6.

An EIT system consists of two parts data acquisition hardware and image acquisition software $[8,9]$. Figure 1 shows the basic block diagram of EIT system comprised of

1. An array of electrodes, attached to the surface of the phantom and the normal saline as a conducting medium

2. A measurement circuit, which applies an alternating current to the electrodes and measures resultant voltage distribution on the surface of the phantom.

3. An image reconstruction algorithm, which reconstructs images of spatial resistivity distribution from the voltage measurements

4. A user interface (display, hardcopy, keyboard, storage facilities, etc.) to enable an easy user access to the image analysis.

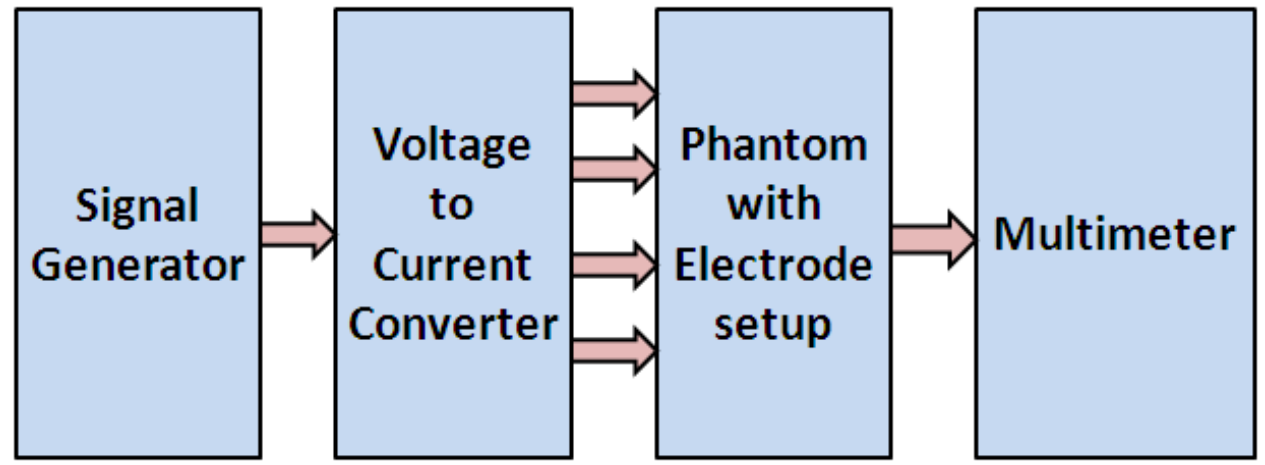

Figure 1. EIT Block Diagram

\section{EIT SYSTEM}

For work mentioned in this paper a similar set has been used. In the present set the voltage output from the signal generator is converted into current. An OP-AMP based voltage to current converter is designed to deliver low value current with variable frequency up to $15 \mathrm{KHz}$. This experiment is performed at $1.3 \mathrm{kHz}$. The experimental set up has 16 electrodes, an alternating current supply and a multimeter for the voltage measurement.

This paper focuses on the EIT experiments performed using normal saline with $0.9 \%$ of sodium chloride in a plastic container (phantom). The diameter of plastic container is $14 \mathrm{~cm}$ and height is 7 $\mathrm{cm}$ and the volume of normal saline used is $360 \mathrm{ml}$. Electrical conductivity of normal saline is 300 $\mathrm{mS} / \mathrm{m}$.

\section{Data Collection}

The 16 electrodes are placed on phantom equidistantly and normal saline is used as a conducting medium. The conducting and non conducting impurities are inserted one after another in the saline solution inside phantom. Here in our experiment the current is applied in bipolar neighbouring configuration but current can also be applied through opposite, cross and adaptive method [2, 7, 9, 10]. The setup of experiment is shown in figure 2 . Although distributed voltage measurements can be made in many ways and patterns, the most common procedure is to measure the voltage difference between adjacent electrodes [1,9]. In this experiment the sets of voltages are obtained by using adjacent pattern. In adjacent pattern the low frequency current is applied to a set of neighbouring electrodes and voltages is measured between remaining set of consecutive electrodes as shown in figure 3. For example, in a set of 16 electrodes the current is applied between electrode pair 1-16 and the voltage is measured between 2-3,3-4 and so on (excluding source pair electrodes). Thus the first 13 readings are obtained. The current source is then shifted to pair 1-2, 2-3...etc and the procedure is repeated to get the next set of 13 readings $[7,9,10]$. Thus by adjacent method 208 (13X16) voltage readings are obtained. All these voltage readings are taken manually and it takes nearly 20 minutes to 
International Journal of Engineering Sciences \& Emerging Technologies, Feb 2012. ISSN: 2231 - 6604 doi: 10.7323/ijeset/v1_i2_10

Volume 1, Issue 2, pp: 83-89 OIJESET

take one set of voltage readings. The non conducting impurity used in this experiment is clay cylinder of $18 \mathrm{~mm}$ diameter and $70 \mathrm{~mm}$ height and conducting impurity used in this experiment is copper spindle of $35 \mathrm{~mm}$ diameter. After taking the voltage readings finite element method is used for reconstruction of images which is explained in next section.

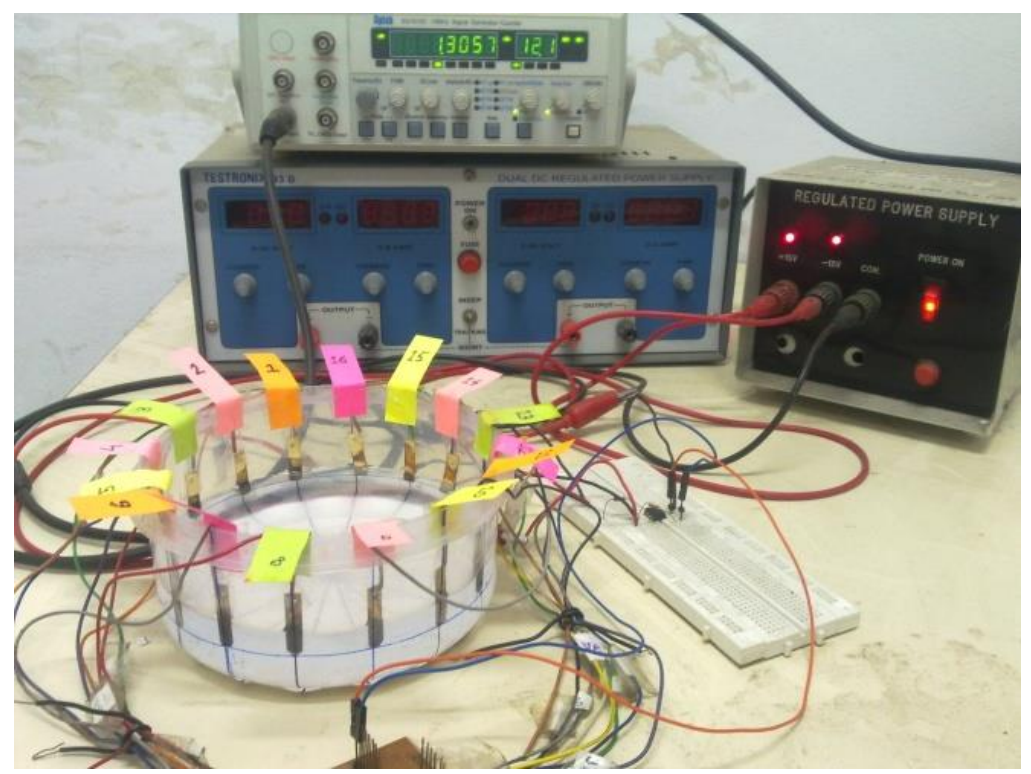

Figure 2. Experimental Setup

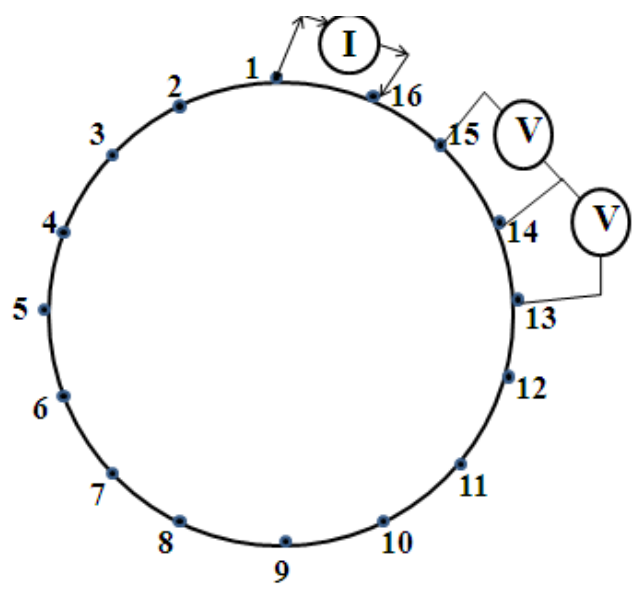

Figure 3. Adjacent Pattern of Measurement

\section{Finite Element Method Reconstruction}

The finite element method has developed simultaneously with the increasing use of high speed computers with the growing emphasis on iterative methods for engineering analysis. Although the method was originally developed for structural analysis, the general nature of the theory on which it is developed, made possible for application in various fields like medical imaging. Problems involving complex material properties like human tissues are solved using numerical methods [11] and these methods do not provide exact but approximate and barely acceptable solutions [12].

Figure 4(a) shows the container filled with normal saline and with no impurity. The density distribution is uniform in this case and can be seen in FEM Graph as shown in figure 4(b).The uniform sunshine yellow colour indicates the almost constant potential in the phantom. The ochre yellow spots show the very small increase in voltage near the electrodes. 
International Journal of Engineering Sciences \& Emerging Technologies, Feb 2012. ISSN: 2231 - 6604 doi: 10.7323/ijeset/v1_i2_10 Volume 1, Issue 2, pp: 83-89 OIJESET

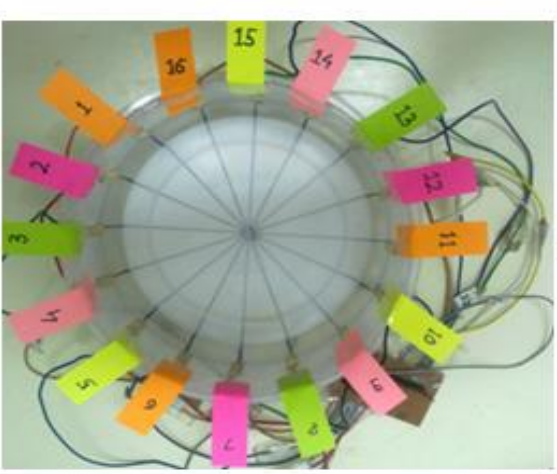

(a)

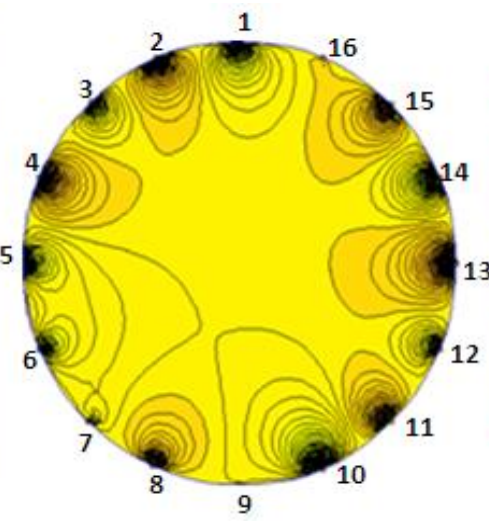

(b)

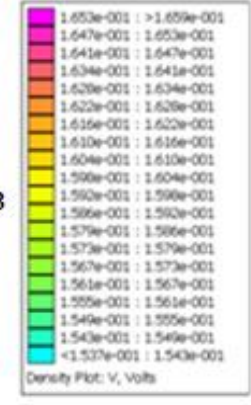

Figure 4. (a) Container without impurity and (b) its voltage density plot

Figure 5(a) shows the container with non-conducting clay cylinder is placed near electrodes 8 and 9 . Figure 5(b) shows the deviation in potential lines. The magenta colour of FEM graph indicates the increase in voltage near electrodes 8 and 9 which confirms the presence of non conducting impurity.

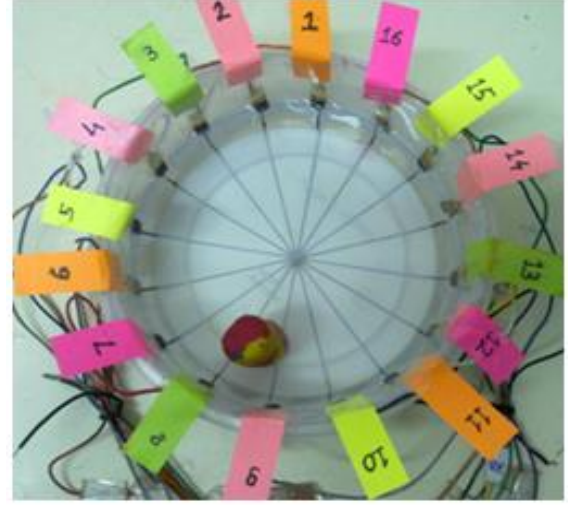

(a)
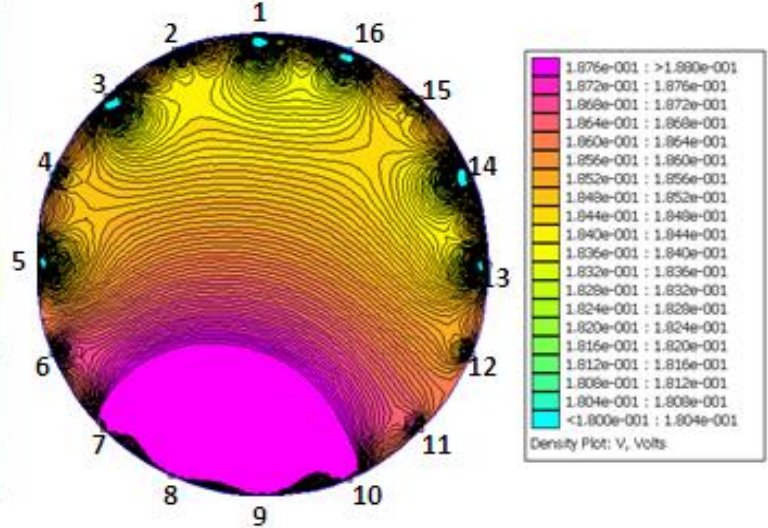

(b)

Figure 5. (a) Container with non-conducting clay near 8 and 9 and (b) its voltage density plot

The position of same clay cylinder is changed to electrodes 4 and 5 as shown in figure 6(a) and the experiment is repeated for this clay position. The figure 6(b) shows the increase in voltage near to electrodes 4 and 5 ensuring the presence of non conducting impurity at that location.

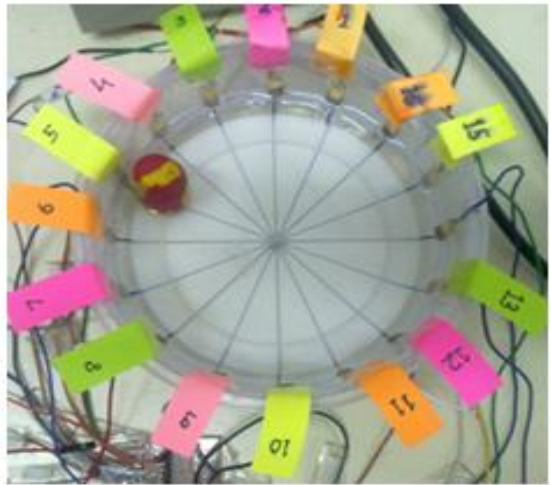

(a)
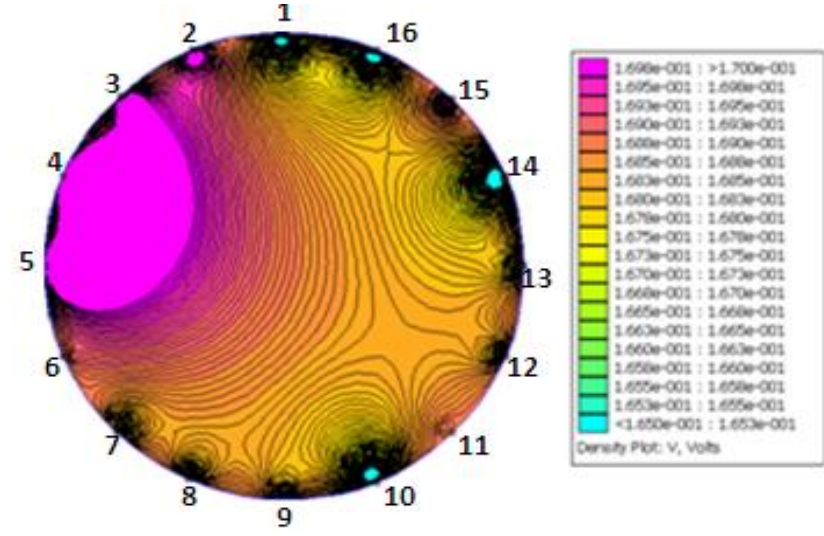

(b)

Figure 6. (a) Container with clay near 4 and 5 and (b) voltage density plot 
International Journal of Engineering Sciences \& Emerging Technologies, Feb 2012. ISSN: 2231 - 6604 doi: 10.7323/ijeset/v1_i2_10

Volume 1, Issue 2, pp: 83-89 OIJESET

The impurity later is changed to conducting copper spindle and the experiment is performed to find its effect on voltage density distribution. Figure 7(a) shows the position of copper spindle and in figure 7(b) FEM graph of potential lines. The cyan colour in FEM graph indicates the decrease in voltage near electrodes 8 and 9 which confirms the presence of conducting impurity.

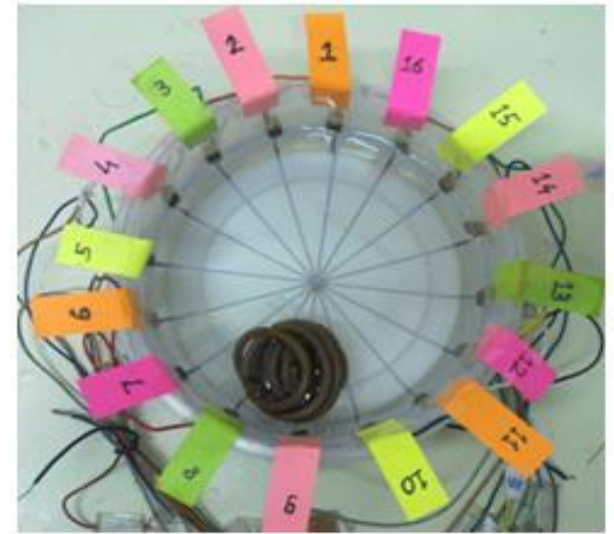

(a)

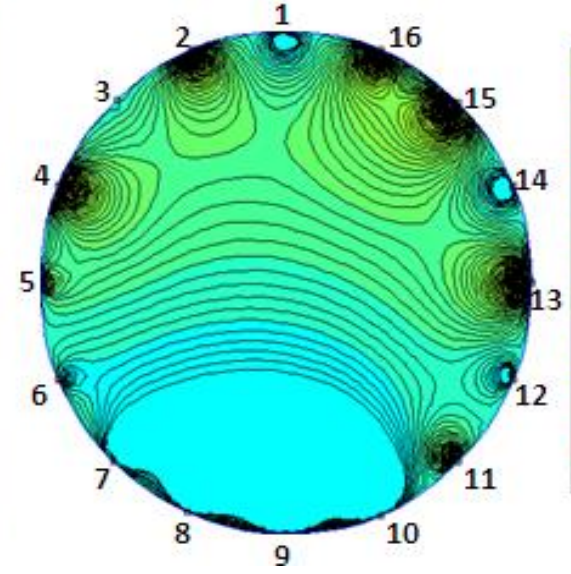

(b)

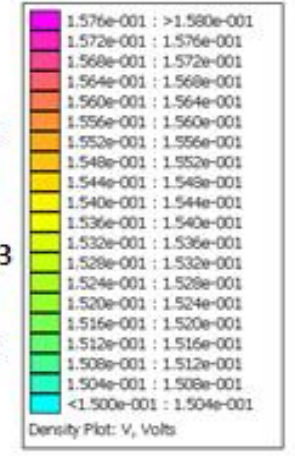

Figure 7. (a) Container with copper spindle near 8 and 9 and (b) voltage density plot

This experiment is repeated by changing the position of impurity to electrode 4 and 5. Figure $8(a)$ shows copper spindle. Figure 8(b) shows the FEM plot of voltage density distribution of the copper spindle near electrode no 4 and 5.

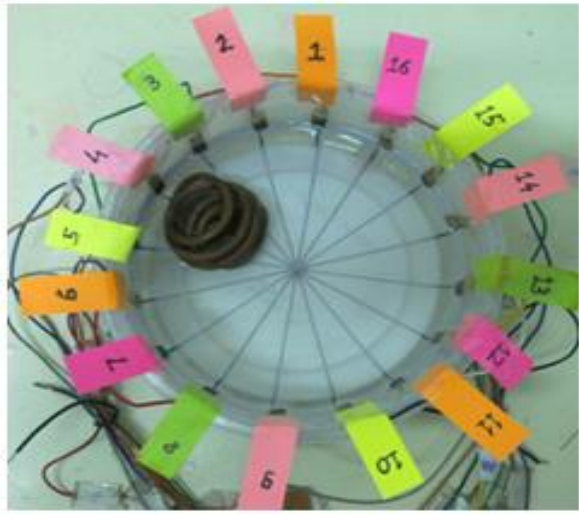

(a)

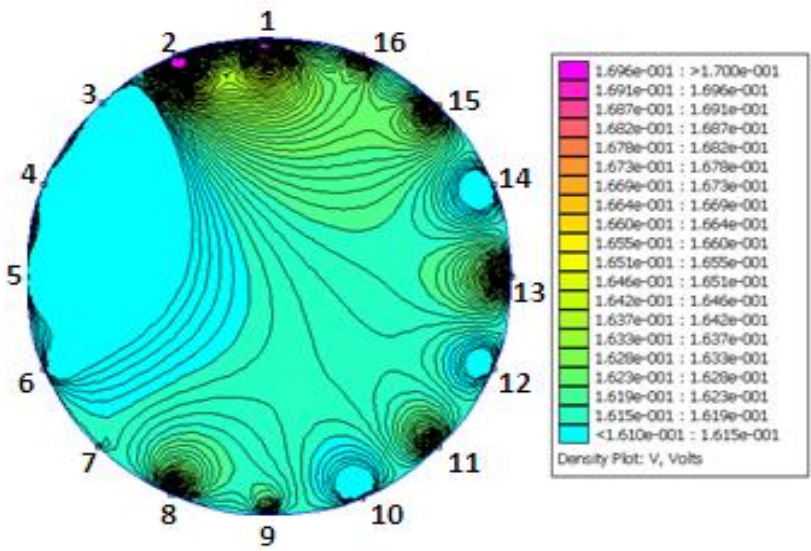

(b)

Figure 8. (a) Copper spindle near electrode 4 and 5 (b) voltage density plot

\section{RESUlt AND DiscusSiON}

First the current source of low value is designed and the EIT system is assembled as shown in figure 2. The two types of impurities of the known area are inserted one after the other in the normal saline and the readings are taken. The superimposed voltages appearing on the surface of phantom are used to reconstruct the impedance images. The behaviour of potential lines in the FEM graph is studied. It is found that when the non-conducting impurity is added to the phantom, the equipotential lines are deviated and leave the non-conducting object. As a result the voltage measured near impurity is high i.e. the conductivity is low. Similarly when the conducting impurity is added the equipotential lines tend to pass through it resulting in low voltage and high conductivity.

The FEM graphs clearly indicate the position also the type of impurity. But the actual size and shape of the impurity cannot be detected. The table 1 shows the actual area of the impurity and the 
International Journal of Engineering Sciences \& Emerging Technologies, Feb 2012. ISSN: 2231 - 6604 doi: 10.7323/ijeset/v1_i2_10 Volume 1, Issue 2, pp: 83-89 OIJESET

reconstructed area respectively. The size of the clay cylinder is $0.002205398 \mathrm{~m}^{2}$ and after 2540 meshes it is calculated as $0.00172318 \mathrm{~m}^{2}$. Similarly the difference in the size of copper spindle is clearly seen.

Table 1. Sizes of impurities real and reconstructed

\begin{tabular}{|l|l|l|}
\hline Impurity Type & Actual size & Reconstruct. size \\
\hline Clay cylinder & $0.002205398 \mathrm{~m}^{2}$ & $0.00170318 \mathrm{~m}^{2}$ \\
\hline Copper spindle & $0.002827433 \mathrm{~m}^{2}$ & $0.00222543 \mathrm{~m}^{2}$ \\
\hline
\end{tabular}

\section{CONCLUSION AND SUMMARY}

The Electrical Impedance Tomography is a no radiation, safe, portable and affordable medical imaging technique. However, the resolution of EIT system is poor. The performance of EIT system can be improved by using more number of electrodes and increasing frequency of the current source. As the size of the phantom circumference is fixed, the size of the electrodes can be kept optimum to achieve best resolution. The colour of FEM graph indicates the presence, the type and the location of the impurity. This technique can be used for various applications such as anomaly detection in human tissue, pulmonary monitoring, hyperthermia monitoring, foetal movement monitoring, imaging of intravascular haemorrhage in infants and foetal imaging.

\section{ACKNOWLEDGEMENT}

The authors thank Xavier Institute of Engineering and VJTI. This work was not possible without the help of Fr Reginald Tauro, Director XIE and Fr. Fabian Barreto, Administrator XIE. Also the authors thank all those who are directly and indirectly involved in the conduction of EIT experiments.

\section{REFERENCES}

[1] Mohd Tahir Erwati, \& Nagi Farrukh, "Applications of electrical impedance tomography for imaging in biomedical and material technology", Proceedings of 2009 IEEE Students Conference on research and development.(SCOReD 2009), 16-18 Nov '09, UPM Serdang, Malaysia.

[2] Sarwan Kumar, Sneh Anand \& Amit Sengupta,'Impedence based image reconstruction of the field distribution inside the closed phantom using finite element method", (IJCNS) International journal of computer and network security. Vol. 2, No 7, July 2010.

[3] Y. Zou \& Z. Guo, "A review of electrical impedance techniques for breast cancer detection" Elsevier Medical Engineering and Physics 25 / (2003).

[4] Jianjun Zhang, Weili Yan, Guizhi Xu \& Quanming Zhao, "A New Algorithm to Reconstruct EIT Images : Node Back Projection Algorithm ," Proceedings of the $20^{\text {th }}$ Annual International conference of IEEE EMBS. Cite Internationale, Lyon, France August 23-26, 2007.

[5] John G. Webster, "Medical Instrumentation Application and Design", third edition, Wiley India, 2007.

[6] R.S. Khandpur, "Handbook of biomedical instrumentation", Second Edition, tata mc Graw hill, pp 670-683.

[7] T. K. Bera \& J. Nagaraju, "Studying the resistivity imaging of chicken tissue phantoms with different current patterns in Electrical Impedance Tomography(EIT)", Measurement(2012), doi: 10.1016/j.measurement.2012.01.002.

[8] Manuchehr Soleimani, "Electrical impedance tomography system: an open access circuit design" Biomedical Engineering Online, o3 May 2006.

[9] B H Brown, R H Smallwood, D C Barber, P V Lawford \& D R Hose, "Medical Science Series MEDICAL PHYSICS AND BIOMEDICAL ENGINEERING" Department of Medical Physics and Clinical Engineering, University of Sheffield and Central Sheffield University Hospitals, Sheffield, UK Institute of Physics Publishing Bristol and Philadelphia(1999), chapter 12.

[10] Prabhakar Manage, Vidya Sarode \& Dr Alice Cheeran, "Electrical Impedance Tomography And Its Applications" i-COST Electronics \& Communication Conference proceedings 13-15 January 2011 S.S.V.P.S‘s B.S. Deore College of Engineering \& Polytechnic, Dhule Page2.22. 1 
International Journal of Engineering Sciences \& Emerging Technologies, Feb 2012. ISSN: 2231 - 6604 doi: 10.7323/ijeset/v1_i2_10 Volume 1, Issue 2, pp: 83-89 OIJESET

[11] Holder DS, “Electrical Impedance Tomography”, IoP Publishing, Bristol, UK, 2004.

[12] Desai \& Abel, “An introduction to finite element method” Affiliated east west press, New Delhi, 1972.

\section{Authors}

Vidya Sarode has completed B. E in Electronics Engineering in 1987 from Amravati University. She joined XIE, Mumbai as an assistant professor in 2006. She completed Masters in Electrical Engg with specialization in Industrial Electronics 1996 from M.S. University Baroda. Further she joined Ph. D. program at VJTI, Mumbai in 2009 in the topic of "Breast Cancer Detection". Currently she is working as an Associate Professor in the Electronics and Telecommunication Engineering department, Xavier Institute of Engineering, Mumbai.

Priya M. Chimurkar has completed B. E in Electronics and Telecommunication Engineering in 2010 from Amravati University. Currently she is pursuing Masters in Electronics and Telecommunication Engineering VJTI, Mumbai.

Alice Cheeran has completed B. E in Electrical Engineering in 1984 from Kerala University. She joined V J T I, Mumbai as a lecturer in 1987. She completed Masters in Electrical Engg with specialization in Control Systems and in Electronics respectively in 1994 and 1996 from Mumbai University. Further she did $\mathrm{Ph}$. D. from IIT Bombay in 2005 in the topic of "Speech Processing for the Hearing Impaired". Currently she is working as an Associate Professor in the Electrical Engineering department, V J T I, Mumbai.
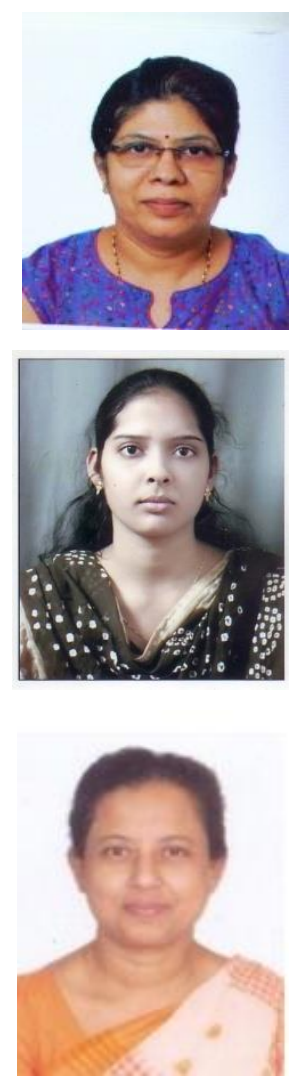